\title{
Polytopes associated to dihedral groups
}

\author{
Barbara Baumeister * \\ Universität Bielefeld, Germany \\ Christian Haase ${ }^{\dagger}$ \\ Goethe-Universität Frankfurt, Germany \\ Benjamin Nill $\ddagger$ \\ Case Western Reserve University, Cleveland, OH, USA \\ Andreas Paffenholz ${ }^{\S}$ \\ Technische Universität Darmstadt, Germany
}

Received 2 December 2011, accepted 30 October 2012, published online 8 January 2013

\begin{abstract}
In this note we investigate the convex hull of those $n \times n$ permutation matrices that correspond to symmetries of a regular $n$-gon. We give the complete facet description. As an application, we show that this yields a Gorenstein polytope, and we determine the Ehrhart $h^{*}$-vector.
\end{abstract}

Keywords: Permutation polytopes, dihedral groups, lattice polytopes.

Math. Subj. Class.: 20B35, 52B12; 05E10, 52B05, 52B20

*The first author likes to thank for the support by the DFG through the SFB 701 "Spectral Structures and Topological Methods in Mathematics".

${ }^{\dagger}$ The second author is supported by DFG Heisenberg (HA 4383/4).

$\ddagger$ The third author is supported in part by the US National Science Foundation (DMS 1203162).

$\S$ The last author is supported by the Priority Program 1489 "Algorithmic and Experimental Methods in Algebra, Geometry and Number Theory" of the German Research Council (DFG).

E-mail addresses: b.baumeister@math.uni-bielefeld.de (Barbara Baumeister), haase@ math.uni-frankfurt.de (Christian Haase), benjamin.nill@ case.edu (Benjamin Nill), paffenholz@mathematik.tu-darmstadt.de (Andreas Paffenholz) 


\section{Introduction}

To any finite group $G$ of real $n \times n$ permutation matrices we can associate the permutation polytope $P(G)$ given by the convex hull of these matrices in the vector space $\mathbb{R}^{n \times n}$. A well-known example of such a polytope is the Birkhoff polytope $B_{n}$, which is defined as the convex hull of all $n \times n$ permutation matrices [9, 8]. This polytope appears in various contexts in mathematics from optimization to statistics to enumerative combinatorics. (See, e.g., $[24,20,21,2,1]$.) It is also a famous example of a Gorenstein polytope (see Section 5). Gorenstein polytopes turn up in connection to mirror symmetry in theoretical physics.

Guralnick and Perkinson [15] studied polytopes associated to general subgroups $G$ of the symmetric group and proved results about their dimension, and about the diameter of their vertex-edge graph. A systematic exposition of general permutation polytopes is given in [5]. There, we studied which groups lead to affinely equivalent polytopes, we considered products of groups and polytopes, classified low-dimensional cases, and we formulated several open conjectures.

In order to get an intuition about what one can expect in general, it is instructive to consider some special classes of permutation groups. A seemingly very difficult case is when $G$ equals the group of even permutation matrices. Just to exhibit exponentially many facets is already a daunting task, for this see [17]. Even for cyclic $G$ we showed in [6] that these polytopes have a surprisingly complex and not yet fully understood facet structure.

In [12] Collins and Perkinson studied polytopes given by Frobenius groups. A special case is the dihedral group $D_{n}$ for $n$ odd, which was considered in more detail by Steinkamp [22]. Since $D_{n}$ is the automorphism group of a regular $n$-gon, the cases where $n$ is even and odd are quite different.

The most recent paper on permutation polytopes [11] focused on determining the volumes of permutation polytopes associated to cyclic groups, dihedral groups, and Frobenius groups. In order to compute the volume of $P\left(D_{n}\right)$, the authors find a Gale dual combinatorial description, which they use to provide an explicit formula for the Ehrhart polynomial of $P\left(D_{n}\right)$.

The dihedral group $D_{n}$ is the automorphism group $\operatorname{Aut}\left(C_{n}\right)$ of a cycle $C_{n}$, and any permutation matrix $M(\sigma)$ of an element $\sigma \in D_{n}$ commutes with the adjacency matrix $A$ of $C_{n}$. So any point in $P\left(D_{n}\right)$ commutes with $A$, and

$$
P\left(D_{n}\right) \subseteq\left\{M \in \mathbb{R}^{n \times n} \mid M \text { is doubly stochastic and } M A=A M\right\} .
$$

Here, a matrix is doubly stochastic if all entries are non-negative and each row and column sum is 1. Tinhofer [24] asks, more generally, for a classification of those undirected graphs $G$ where the two sets above are equal, i.e. where the commutation condition $M A=A M$ already suffices to characterize the elements of $P(\operatorname{Aut}(G))$ among all doubly stochastic matrices. The Birkhoff-von Neumann theorem is the special case where $A$ is the unit matrix. Tinhofer shows that this also holds for the adjacency matrices of cycles and trees [24, Theorems 2\&3].

In this note, we independently investigate $P\left(D_{n}\right)$ in a more direct and elementary way. We give a complete list of its facet inequalities (Theorem 3.3, Theorem 4.1). As an application, we observe that these lattice polytopes are Gorenstein polytopes, and we get a nice description of the generating function of their Ehrhart polynomials (Theorem 5.3, Corollary 5.4).

Acknowledgments: Many results are based upon experiments and computations using 
the package polymake [14] by Gawrilow and Joswig. We would like to thank the referees for carefully reading and improving the text.

\section{Notation and preliminary results}

Let $S_{n}$ be the permutation group on $n \geq 3$ elements. Every permutation $\sigma \in S_{n}$ can be represented by an $n \times n$ matrix $M_{\sigma}$ with entries $\delta_{i,(j) \sigma}$. So the entries are in $\{0,1\}$ and there is exactly one 1 in each row and column. Notice that we apply matrices and permutations from the right. We can view such a matrix as a vector in $\mathbb{R}^{n^{2}}$. For a subgroup $G$ of $S_{n}$ we define the polytope

$$
P_{G}:=\operatorname{conv}\left(M_{\sigma} \mid \sigma \in G\right) .
$$

This is a 0/1-polytope, so all matrices are in fact vertices of the polytope.

We denote by $D_{n}$ the subgroup of $S_{n}$ corresponding to the symmetry group of the regular $n$-gon, the dihedral group of order $2 n$. This group is generated by two elements. If $n$ is odd, then these may taken to be the rotation $\rho$ of the $n$-gon by $360 / n$ degrees, and the reflection $\tau$ along a line through one vertex and the midpoint of the opposite edge. If $n$ is even, then the second generator $\tau$ is instead the reflection along a line through two opposite vertices. Thus $\rho$ is the permutation $(1,2, \ldots, n)$ and $\tau$ the reflection $(2, n)(3, n-$ $1) \cdots((n+1) / 2,(n+3) / 2)$ if $n$ is odd and $(2, n)(3, n-1) \cdots(n / 2,(n / 2)+2)$ if $n$ is even.

The associated permutation polytope is the convex hull of the corresponding matrices,

$$
\operatorname{DP}_{n}:=\operatorname{conv}\left(M_{\sigma} \mid \sigma \in D_{n}\right) .
$$

The dihedral group $D_{n}$ has $2 n$ elements

$$
\rho^{0}, \rho^{1}, \rho^{2}, \ldots, \rho^{n-1}, \tau, \tau \rho, \tau \rho^{2}, \tau \rho^{3}, \ldots, \tau \rho^{n-1} .
$$

We label the vertices of $\mathrm{DP}_{n}$ by $v_{0}, \ldots, v_{n-1}, w_{0}, \ldots, w_{n-1}$ in this order. Let us give a more convenient way to write these matrices.

Let $I$ be the $n$-dimensional identity matrix and $R$ be the $n \times n$ matrix that has 0 's everywhere except at the $n$ entries $(i, j)$, where $0 \leq i, j \leq n-1$ and $j \equiv i+1 \bmod n$ :

$$
R=\left[\begin{array}{ccccccc}
0 & 1 & 0 & 0 & \cdots & 0 & 0 \\
0 & 0 & 1 & 0 & \cdots & 0 & 0 \\
\vdots & \ddots & \ddots & \ddots & & \vdots & \vdots \\
\vdots & & \ddots & \ddots & \ddots & \vdots & \vdots \\
0 & \cdots & \cdots & 0 & \cdots & 1 & 0 \\
0 & 0 & 0 & 0 & \cdots & 0 & 1 \\
1 & 0 & 0 & 0 & \cdots & 0 & 0
\end{array}\right]
$$

Reading the matrices $M_{\sigma}$ row by row, we can identify $M_{\sigma}$ with a (row) vector in $\mathbb{R}^{n^{2}}$. For instance, the $2 \times 2$ identity matrix would be identified with $\left(\begin{array}{llll}1 & 0 & 0 & 1\end{array}\right)$. Under this identification the vertices of $\mathrm{DP}_{n}$ are (in the order given above) the rows of the $2 n \times n^{2}$ matrix

$$
\left[\begin{array}{ccccc}
R^{0} & R^{1} & R^{2} & \cdots & R^{n-1} \\
R^{0} & R^{-1} & R^{-2} & \cdots & R^{-(n-1)}
\end{array}\right]
$$


Permuting the coordinates (corresponding to a linear automorphism of $\mathbb{R}^{n^{2}}$ ) we may write the vertices in the form

$$
V=\left[\begin{array}{ccccc}
I & I & I & \cdots & I \\
I & R^{-2} & R^{-4} & \cdots & R^{-2(n-1)}
\end{array}\right] .
$$

Clearly, the first $2 n$ coordinates of the vertices linearly determine the remaining coordinates. So we can project onto $\mathbb{R}^{2 n}$ without changing the combinatorics of the polytope. Hence, we observe that the dimension of $\mathrm{DP}_{n}$ is at most $2 n$.

\section{The situation for odd $n$}

In this section we completely describe $\mathrm{DP}_{n}$ for $n$ odd. As it will turn out, it is useful to introduce a new polytope that will serve as a basic building block for both situations of even $n$ and odd $n$.

Definition 3.1. Let $Q_{n}$ be the polytope defined as the convex hull of the rows of the $2 n \times n^{2}$ matrix

$$
W:=\left[\begin{array}{lllll}
I & I & I & \ldots & I \\
I & R^{1} & R^{2} & \ldots & R^{n-1}
\end{array}\right] .
$$

While $Q_{n}$ differs from $\mathrm{DP}_{n}$ for even $n$, for odd $n$ the $R^{2 k}$ for $0 \leq k \leq n-1$ are a permutation of the $R^{k}$ for $0 \leq k \leq n-1$. So we deduce from (2.1) that, for $n$ odd, $Q_{n}$ is up to a permutation of coordinates just the polytope $\mathrm{DP}_{n}$.

Proposition 3.2. For odd $n$, the polytopes $\mathrm{DP}_{n}$ and $Q_{n}$ are affinely isomorphic.

The following theorem examines the structure of $Q_{n}$ for arbitrary $n$. For $n$ odd, this result is a special case of Theorem 4.4 in [12].

Let us fix some convenient notation. We denote by $\Delta_{r}$ the $r$-dimensional simplex. We also use for any two integers $s, k$, the term $[s]_{k} \in\{0, \ldots, k-1\}$ to denote the remainder of $s$ upon division by $k$. The free sum of two polytopes $P$ and $P^{\prime}$ of dimensions $d$ and $d^{\prime}$ is the polytope

$$
P \oplus P^{\prime}:=\operatorname{conv}\left(\left\{(p, 0) \in \mathbb{R}^{d+d^{\prime}} \mid p \in P\right\} \cup\left\{\left(0, p^{\prime}\right) \in \mathbb{R}^{d+d^{\prime}} \mid p^{\prime} \in P^{\prime}\right\}\right) .
$$

Theorem 3.3 (Collins\&Perkinson [12]). Let $n$ be odd or even. The polytope $Q_{n}$ has dimension $2 n-2$ and is a free sum of two copies of $\Delta_{n-1}$. Taking coordinates $x_{0}, \ldots, x_{n^{2}-1}$ for $\mathbb{R}^{n \times n}$, its affine hull is given by the equations

$$
\begin{aligned}
& 1=\sum_{i=l n}^{(l+1) n-1} x_{i} \\
& 0=x_{k n+[j]_{n}}-x_{(k+1) n+[j]_{n}}-x_{(k+1) n+[j+1]_{n}}+x_{(k+2) n+[j+1]_{n}}
\end{aligned}
$$

for $0 \leq l \leq n-1,0 \leq j \leq n-2,0 \leq k \leq n-3$.

An irredundant system of inequalities defining the polytope inside its affine hull is given by the inequalities

$$
x_{i} \geq 0
$$

for $0 \leq i \leq n^{2}-1$. 
Proof. All the given equations are satisfied by the vertices of $Q_{n}$. There are $n$ equations of type (aff) and $n^{2}-3 n+2$ equations of type $\left(A_{j, k}\right)$. They are easily seen to be linearly independent, so the dimension of $Q_{n}$ is at most $2 n-2$. On the other hand, deleting any row of $W$ leaves us with a linearly (and hence affinely) independent set of row vectors. (Observe that deleting a row leaves us with a column that contains exactly one 1.) Hence, $\operatorname{dim}\left(Q_{n}\right)=2 n-2$ and the given equations define the affine hull of $Q_{n}$ in $\mathbb{R}^{n^{2}}$.

Further, we see that every $2 n-1$ of the $2 n$ rows of $W$ span the affine hull of $Q_{n}$. So any facet of $Q_{n}$ has $2 n-2$ vertices. Since the inequalities $x_{j} \geq 0$ are 0 on exactly $2 n-2$ of the rows, they all define facets.

In order to prove that $Q_{n}$ is a free sum of simplices we observe that the first $n$ and the last $n$ vertices define $(n-1)$-dimensional simplices sitting in transversal subspaces (intersecting in the matrix corresponding to the row vector $(1 / n, \ldots, 1 / n))$. Therefore, the combinatorial dual of $Q_{n}$ corresponds to the product of $\Delta_{n-1}$ with itself. In particular, $Q_{n}$ has precisely $n^{2}$ facets, so the facet description given above is complete.

\section{The situation for even $n$}

Recall that the join $P \star Q$ of two polytopes $P$ and $Q$ is the convex hull of $P \cup Q$ after embedding $P$ and $Q$ in skew affine subspaces. The dimension of $P \star Q$ equals $\operatorname{dim}(P)+$ $\operatorname{dim}(Q)+1$. For instance, the join of two intervals is a tetrahedron.

Theorem 4.1. Let $n$ be even. The polytope $\mathrm{DP}_{n}$ is a join of two copies of $Q_{n / 2}$. In particular, its dimension is $2 n-3$.

Combined with Theorem 3.3, this result gives a complete description of the facet inequalities and the affine hull equations of $\mathrm{DP}_{n}$ for $n$ even.

Proof. Permuting the coordinates, we can transform $V$ (see (2.1)) into

$$
\left[\begin{array}{cccccccccc}
I & I & I & \cdots & I & I & I & I & \cdots & I \\
R^{0} & R^{2} & R^{4} & \cdots & R^{n-2} & R^{0} & R^{2} & R^{4} & \cdots & R^{n-2}
\end{array}\right] .
$$

Clearly, projecting onto the first $\frac{n^{2}}{2}$ coordinates yields an affine isomorphism of $\mathrm{DP}_{n}$ onto the convex hull of the rows of the $2 n \times \frac{n^{2}}{2}$ matrix

$$
\left[\begin{array}{lllll}
I & I & I & \ldots & I \\
R^{0} & R^{2} & R^{4} & \ldots & R^{n-2}
\end{array}\right]
$$

In the representation given by this matrix let us partition the set of $2 n$ vertices (labelled from 0 to $2 n-1$ ) into two sets: consisting of the $n$ rows with even index and the $n$ rows with odd index.
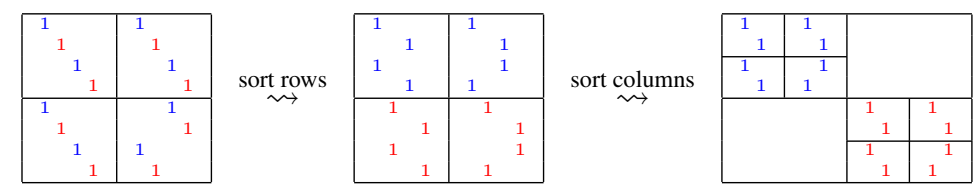

Then we permute the $\frac{n^{2}}{2}$ coordinates in such a way that in the first set of rows (corresponding to even vertices) all nonzero entries are in the first half (i.e. in the first $\frac{n^{2}}{4}$ columns). 
Then all nonzero entries in the second set of rows (corresponding to the odd vertices) will be in the second half (i.e. in the last $\frac{n^{2}}{4}$ columns). By a permutation of the coordinates within the first half we get that the rows of even vertices yield precisely the vertex set of $Q_{n / 2} \times\{0\}$ (for $0 \in \mathbb{R}^{\frac{n^{2}}{4}}$ ). In the same way, the coordinates in the second half can be permuted so that the rows of odd vertices equal the vertices of $\{0\} \times Q_{n / 2}$ (for $0 \in \mathbb{R}^{\frac{n^{2}}{4}}$ ). Since 0 is not in the affine hull of $Q_{n / 2}$, we deduce that $\mathrm{DP}_{n}$ is a join of two copies of $Q_{n / 2}$. Hence, its dimension equals $2 \operatorname{dim}\left(Q_{n / 2}\right)+1=2(n-2)+1=2 n-3$ by Theorem 3.3.

\section{Lattice properties}

$\mathrm{DP}_{n}$ and $Q_{n}$ are lattice polytopes, i.e. their vertices lie in the lattice $\mathbb{Z}^{n^{2}}$ of integral vectors. It is readily checked that all above affine isomorphisms respect lattice points. In this section, we will show that these lattice polytopes have especially nice properties which allow us to completely describe their Ehrhart $h^{*}$-vectors.

A $d$-dimensional lattice polytope $P$ containing 0 in its interior is reflexive, if its polar (or dual) polytope

$$
P^{*}:=\left\{x \in \mathbb{R}^{d} \mid\langle x, v\rangle \geq-1 \forall v \in P\right\}
$$

is again a lattice polytope (in the dual lattice). This notion was introduced by Batyrev in [3]. A generalization of this is the class of Gorenstein polytopes. A lattice polytope is a Gorenstein polytope of codegree $k$, if there is a positive integer $k$ and an interior lattice point $m$ in $k P$ such that $k P-m$ is a reflexive polytope. Such polytopes play an important role in the classification of Calabi-Yau manifolds for string theory. See [4] for basic properties. The next proposition tells us that the polytopes $Q_{n}$ belong to this class. The normalized volume of $\mathbb{R}^{n}$ is the volume form which assigns to the standard simplex the volume 1 .

Proposition 5.1. Let $n$ be odd or even. The polytope $Q_{n}$ is Gorenstein of codegree $n$ and normalized volume $n$.

Proof. By Theorem 3.3, the point $\frac{1}{n}(1,1, \ldots, 1)$ is an interior point of $Q_{n}$ with equal integral distance $1 / n$ to all facets, and $m:=(1,1, \ldots, 1)$ is the unique interior lattice point in $n Q_{n}$. Hence $n Q_{n}-m$ is a reflexive polytope.

By Theorem 3.3, all facets of $Q_{n}$ are simplices of facet width 1 , hence they are all unimodular. As we have seen, multiplying by $n$ gives (up to translation) a reflexive polytope with the unique interior lattice point $m=(1,1, \ldots, 1)$. The normalized volume of $n Q_{n}$ is the sum of the volumes of $n^{2}$ pyramids over facets with apex $m$. But in $n Q_{n}$ each facet has normalized volume $n^{2 n-3}$, and the apex has lattice distance 1 from the facet, so each pyramid has normalized volume $n^{2 n-3}$. There are $n^{2}$ of these pyramids, so the normalized volume of $n Q_{n}$ equals $n^{2 n-1}$. Dividing by $n^{2 n-2}$ to get from $n Q_{n}$ back to $Q_{n}$ gives the normalized volume $n$ of $Q_{n}$.

A polytope $P$ is compressed if every so-called pulling triangulation is regular and unimodular. Equivalently, $P$ is compressed if for any supporting inequality $a^{t} x \leq b$ with a primitive integral normal $a$, i.e. with a normal vector whose entries are integers and which is not an integral multiple of some other integer vector, the polytope is contained in the set $\left\{x \mid b-1 \leq a^{t} x \leq b\right\}$. For a more detailed explanation of these terms we refer to [13]. 
This property has strong implications on the associated toric ideal, see e.g. [23]. The next proposition follows immediately from Theorem 1.1 of [19] and Theorem 3.3.

Proposition 5.2. Let $n$ be odd or even. The polytope $Q_{n}$ is compressed.

The Ehrhart polynomial $L_{P}(k):=\left|k P \cap \mathbb{Z}^{d}\right|$ of a $d$-dimensional lattice polytope counts the number of integral points in integral dilates of $P$. It is well known that the generating function of $L_{P}$ is given by

$$
\sum_{m \geq 0} L_{P}(m) t^{m}=\frac{h^{*}(t)}{(1-t)^{d+1}}
$$

for some polynomial $h^{*}$ of degree at most $d$ with integral non-negative coefficients, see [7]. Hence, determining the Ehrhart polynomial is equivalent to finding the $h^{*}$-vector (also called the $\delta$-vector) of coefficients of $h^{*}(t)$. As is well-known, $P$ is Gorenstein if and only if the $h^{*}$-vector is symmetric. The following theorem shows that in our case this vector has a particularly nice form.

Theorem 5.3. Let $n$ be odd or even. The $h^{*}$-vector of $Q_{n}$ satisfies $h_{i}^{*}=1$ for $0 \leq i \leq n-1$ and $h_{i}^{*}=0$ otherwise.

Proof. Since the codegree of $Q_{n}$ is $n$ and its dimension is $2 n-2$ by Theorem 3.3, the maximal non-zero entry of the $h^{*}$-vector has to be $h_{n-1}^{*}$, see [7]. By a theorem of Bruns and Römer [10] we know that the $h^{*}$-vector of a Gorenstein polytope that has a regular unimodular triangulation is symmetric and unimodal. In particular, $h_{i}^{*} \geq 1$ for $i=0, \ldots, n-1$. Since by Proposition 5.1 the sum of the entries of the $h^{*}$-vector equals $n$, the statement follows.

In particular, if $n$ is odd, the previous result describes the $h^{*}$-vector of $\mathrm{DP}_{n}$. Finally, let us deal with the even case.

Corollary 5.4. Let $n$ be even. The $h^{*}$-vector of $\mathrm{DP}_{n}$ equals

$$
\left(1,2,3, \ldots, \frac{n}{2}-1, \frac{n}{2}, \frac{n}{2}-1, \ldots, 2,1\right) .
$$

In particular, the polytope $\mathrm{DP}_{n}$ is Gorenstein of codegree $n$ and normalized volume $n^{2} / 4$.

Proof. By the proof of Theorem 4.1, $\mathrm{DP}_{n}$ is given up to coordinate permutation as the convex hull of the rows of

$$
\left[\begin{array}{ll}
\tilde{W} & 0 \\
0 & \tilde{W}
\end{array}\right]
$$

where $\tilde{W}$ is the $n \times\left(\frac{n}{2}\right)^{2}$ matrix whose rows are the vertices of $Q_{\frac{n}{2}}$ as given in (3.1). The integral linear functional which sums the first $\frac{n}{2}$ coordinates evaluates to 1 on the first $\frac{n}{2}$ rows, and to 0 on the second half. Hence, the two copies of $Q_{\frac{n}{2}}$ (say, $P_{1} \times\{0\}$ and $\{0\} \times P_{2}$ ) have lattice distance 1 in the lattice $\mathbb{Z}^{\frac{n^{2}}{2}} \cap$ aff $\mathrm{DP}_{n}$. In other words, there is an affine isomorphism respecting lattice points which maps $\mathrm{DP}_{n}$ onto the convex hull of $P_{1} \times\{0\} \times\{1\}$ and $\{0\} \times P_{2} \times\{0\}$ in $\mathbb{R}^{\frac{n^{2}}{2}+1}$. Therefore, the statement follows from the well-known fact [7, Example 3.32] that in this case the $h^{*}$-polynomial equals the product of the $h^{*}$-polynomials of $P_{1}$ and $P_{2}$. 


\section{Substructures}

In [5] the authors discussed which subgroups of a permutation group yield faces of $P(G)$. An obvious class of such subgroups are stabilizers:

Take a partition $[n]:=\{1, \ldots, n\}=\bigsqcup I_{i}$. Then the polytope of the stabilizer of the subsets $I_{i}$

$$
\operatorname{stab}\left(G ;\left(I_{i}\right)_{i}\right):=\left\{\sigma \in G \mid \sigma\left(I_{i}\right)=I_{i} \text { for all } i\right\} \leq G
$$

is a face of $P(G)$. The authors conjecture that there are no other examples.

Conjecture 5.8 [5] Let $G \leq S_{n}$. Suppose $H \leq G$ is a subgroup such that $P(H) \preceq P(G)$ is a face. Then $H=\operatorname{stab}\left(G ;\left(I_{i}\right)_{i}\right)$ for a partition $[n]=\bigsqcup I_{i}$.

We have verified the conjecture for $G=S_{n}$ as well as for cyclic subgroups $G \leq S_{n}$, see Proposition 5.9 of [5]. Meanwhile Jessica Nowack and Daniel Heinrich studied this question for the dihedral groups in their Diploma theses.

Proposition 6.1. (Heinrich, Nowack [16, 18]) Conjecture 5.8 holds for $G=D_{n} \leq S_{n}$ for every $n$.

Sketch of the proof. For $n$ odd Heinrich first shows that, if $H$ is the subgroup of all rotations of $G$, then $P_{H}$ is not a face of $P_{G}$. The remaining subgroups are precisely the stabilizers of their orbits, see Theorem 7.1.1 of [16].

For $n$ even the main work is to show that the subgroup of all rotations, the subgroup of the squares of the rotations and finally the subgroup generated by the squares of the rotations and by the reflections through two edges are precisely those subgroups $H$ of $G$ for which $P_{H}$ is not a face of $P_{G}$. Nowack shows that the remaining subgroups are precisely the stabilizers of their orbits, see Section 4.2 of [18].

\section{References}

[1] C. A. Athanasiadis, Ehrhart polynomials, simplicial polytopes, magic squares and a conjecture of Stanley, J. Reine Angew. Math. 583 (2005), 163-174.

[2] A. Barvinok and T. Stephen, The distribution of values in the quadratic assignment problem, Math. Oper. Res. 28(1) (2003), 64-91.

[3] V. V. Batyrev, Dual polyhedra and mirror symmetry for Calabi-Yau hypersurfaces in toric varieties, J. Algebraic Geom. 3(3) (1994), 493-535.

[4] V. Batyrev and B. Nill, Combinatorial aspects of mirror symmetry, in: M. Beck et al. (eds.), Integer points in polyhedra-geometry, number theory, algebra, optimization, statistics, Proceedings of the AMS-IMS-SIAM joint summer research conference (Contemporary Mathematics 452), Snowbird, UT, USA, June 11-15, 2006, American Mathematical Society, Providence, RI, 2008, 35-66.

[5] B. Baumeister, C. Haase, B. Nill and A. Paffenholz, On permutation polytopes, Adv. Math., 222(2) (2009), 431-452.

[6] B. Baumeister, C. Haase, B. Nill and A. Paffenholz, Permutation polytopes of cyclic groups, September 2011, preprint, arXiv:1109.0191.

[7] M. Beck and S. Robins, Computing the continuous discretely, Undergraduate Texts in Mathematics, Springer, New York, 2007. 
[8] L. J. Billera and A. Sarangarajan, The combinatorics of permutation polytopes, in: L. J. Billera et al. (eds.), Formal power series and algebraic combinatorics, Séries formelles et combinatoire algébrique 1994, Invited lectures presented at the 6th international DIMACS workshop, May 23-27, 1994, DIMACS, Ser. Discrete Math. Theor. Comput. Sci. 24, American Mathematical Society, Providence, RI, 1996, 1-23.

[9] R. A. Brualdi and P. M. Gibson, Convex polyhedra of doubly stochastic matrices. I: Applications of the permanent function, J. Comb. Theory, Ser. A 22, (1977) 194-230.

[10] W. Bruns and T. Römer, $h$-vectors of Gorenstein polytopes, J. Comb. Theory, Ser. A 114(1) (2007), 65-76.

[11] K. Burggraf, J. De Loera and M. Omar, On volumes of permutation polytopes, 2011, preprint, arXiv:1103.0039.

[12] J. Collins and D. Perkinson, Frobenius polytopes, January 2004, preprint arXiv: 1102.0988.

[13] J. A. De Loera, J. Rambau and F. Santos, Triangulations. Structures for algorithms and applications, Algorithms and Computation in Mathematics 25, Springer, Berlin, 2010.

[14] E. Gawrilow and M. Joswig, polymake: a framework for analyzing convex polytopes, in: Polytopes—combinatorics and computation (Oberwolfach, 1997), DMV Sem., vol. 29, Birkhäuser, Basel, 2000, 43-73.

[15] R. M. Guralnick and D. Perkinson, Permutation polytopes and indecomposable elements in permutation groups, J. Comb. Theory, Ser. A 113(7) (2006), 1243-1256.

[16] D. Heinrich, Beweis der Seitenvermutung für Permutationspolytope vom Type $D_{2 n}$ mit ungeradem n, Diploma thesis, FU Berlin, October 2011.

[17] J. Hood and D. Perkinson, Some facets of the polytope of even permutation matrices, Linear Algebra Appl. 381 (2004), 237-244.

[18] J. Nowack, Beweis der Seitenvermutung für Permutationspolytope vom Typ $D_{2 n}$ mit geradem $n$, Diploma thesis, FU Berlin, Mai 2011.

[19] H. Ohsugi and T. Hibi, Convex polytopes all of whose reverse lexicographic initial ideals are squarefree, Proc. Am. Math. Soc. 129(9) (2001), 2541-2546.

[20] S. Onn, Geometry, complexity, and combinatorics of permutation polytopes, J. Comb. Theory, Ser. A 64(1) (1993), 31-49.

[21] I. Pak, Four questions on Birkhoff polytopes, Ann. Comb. 4(1) (2000), 83-90.

[22] H. Steinkamp, Convex polytopes of permutation matrices, Bachelor thesis, The Division of Mathematics and Natural Sciences, Reed College, 1999.

[23] B. Sturmfels, Gröbner bases and convex polytopes, University Lecture Series (vol. 8), American Mathematical Society, Providence, RI, 1996.

[24] G. Tinhofer, Graph isomorphism and theorems of Birkhoff type, Computing 36 (1986), 285300. 